\title{
Parallel Machine Scheduling with WMSD Risk Considerations
}

\author{
Ercan Şenyiğgit ${ }^{*}$, Uğur Atıc1² \\ ${ }^{1}$ Erciyes University, Engineering Faculty, Industrial Engineering Department, Kayseri, Turkey (ORCID: 0000-0002-9388-2633) \\ (This publication has been presented orally at HORA congress.)
}

(First received 1 August 2019 and in final form 25 October 2019)

(DOI: 10.31590/ejosat.638286)

\begin{abstract}
ATIF/REFERENCE: Şenyiğit, E. \& Atıcı, U. (2019). Parallel Machine Scheduling with WMSD Risk Considerations. European
\end{abstract} Journal of Science and Technology, (Special Issue), 336-342.

\begin{abstract}
This paper introduces the parallel machine scheduling problems with work related musculoskeletal disorder (WMSD) risk considerations. By the WMSD consideration, we mean that job processing times are an increasing function of occupational repetitive technical actions (OCRA) risk factor. The OCRA index was recruited for risk assessment of occupational repetitive technical actions. WMSD risks were modeled with cumulative mean value of OCRA index. Due to NP-Hard structure of parallel machine scheduling problems with WMSD considerations and learning rate, the solution cannot be found always. However, problem can be solved by transforming assignment problem. In spite of the fact that the computational effort remains $O\left(n^{4}\right)$, problem is solved within more efficient time. In this study, a model that includes learning effect and WMSD risk was proposed. WMSD risk was considered as cumulative mean of risk value throughout shift. In order to the balance between productivity and WMSD risk, jobs' foreseeable cycle time (FCT) value was changed. It is aimed to decrease mean of risk along schedule without increasing total basic process time. The value of sacrifice from the FCT was compensating from job which have optimal, acceptable, borderline or slight risk level. Process time and risk values were recalculated by using new FCT value. Thus, balanced process times and risk values obtained. Proposed model was solved with Lingo software and sequence of jobs was obtained. Total flow time and mean of risk were compared for balanced and none balanced schedules. Total flow time and mean of risk belong to balanced schedule is smaller than none balanced schedule. It was shown that handled problem is solvable at the polynomial time and total flow time can be improved by bringing balance between WMSD risks and productivity.
\end{abstract}

Keywords: scheduling, learning effect, work-related musculoskeletal disorder, OCRA index

\section{Introduction}

The risk assessment in a manufacturing line is done as legal obligation nowadays. It is performing within the scope of occupational health safety. Precautions should be considered towards protection of employees or workplace from possible risks. Risk assessments are similarly used by machine manufacturers and designers for safety in machines and evaluation of human physical performance. However the effect of risk exposed in manufacturing line towards scheduling, processing time and due-dates has not been enlightened yet. In this study it is accepted that, the risks have effects on scheduling, processing time and due-dates, are WMSD risks.

In the literature, the effect of WMSD risk factors depending on repetitive number in scheduling problem has not inspected although the effect of ergonomic risks depending on repetitive number of various occupational diseases or work related musculoskeletal disorders (WMSD) was studied by many scientists.

In the literature, risks in workplaces are generally researched as work related musculoskeletal disorders (WMSD). Minimizing WMSD risks are important in terms of providing sustainable labor force and productivity. Furthermore WMSD risk assessments are mandatorily done within the scope of occupational health and safety legislation and national/international standards (TR, 2012; TSE, 2011; Şenyiğit \& Atici, 2018). Risk assessment and management were established in scientific field 30-40 years ago and many developments were performed in theoretical field (Aven, 2016).

Nowadays WMSD risks become applicable and searchable in many industrial fields. Employees in different business sector from building trade to fishery industry are exposed to the risks, which has been searched. Antwi-Afari et al. worked on risk assessment involving construction workers creating repetitive lifting action. It is reported that workers are exposed to risk factors such as lifting

* Corresponding Author: Erciyes University, Engineering Faculty, Industrial Engineering Department, Kayseri, Turkey, ORCID: 0000-0002-93882633, senyigit@erciyes.edu.tr 
posture during lifting period, lifting weight and repetitive lifting actions, have higher risk of developing WMSD. It is also reported that defining risk factors towards increasing occupational health and sufficiency contributes to understand WMSD risk assessment methods. (Antwi-Afari et al., 2017). Fulmer et al. researched musculoskeletal disorders (MSD) risks among crab hunters in North America. It is stated that MSD prevails $82 \%$ among crab hunters and it has effect on skills of employees (Fulmer, Buchholz, Scribani, \& Jenkins, 2017). Battini et al. held ergonomic risk factors in deciding lot-sizing. It is also reported that material handling has an important effect in deciding lot-sizing. It is evaluated with taking risk factors into consideration in taking of lot-sizing decision decrease developing occupational disease risk and it increases employee performance (Battini, Glock, Grosse, Persona, \& Sgarbossa, 2015). Rapid upper limb assessment (RULA), Rapid Entire Body Assessment (REBA) and The European Assembly Worksheet (EAWS) methods present score as output (McAtamney \& Nigel Corlett, 1993); (Hignett \& McAtamney, 2000); (Schaub, Caragnano, Britzke, \& Bruder, 2013). OCRA (Occupational Repetitive Actions) index is one of the methods that is used for risk assessment of musculoskeletal disorders regarding repetitive technical activities (Occhipinti, 1998). OCRA index provides valid index value based on observation associated with pathologic case percentage expected among all working population (Micheli \& Marzorati, 2018; Şenyiğit \& Atici, 2018).

A checklist was also formed towards risk assessment of WMSD. It is reported that the relation between the score obtained as a result of checklist and OCRA index, was taken into account. Criteria, procedure and risk assessment tool was formed towards protection from WMSDs and analysis of biomechanical loads (Occhipinti \& Colombini, 2016). A heuristic model in OCRA index for solution of assembly line balancing was proposed (Otto \& Scholl, 2011). Boenzi et al. presented OCRA based approach to job rotation schedules and optimal breaks in assembly line. It was proposed two different integer programming models. Work load risk and acceptability were evaluated by using OCRA index (Boenzi, Digiesi, Mossa, Mummolo, \& Romano, 2013). The assembly line balancing problem under ergonomic risk factors was presented. OCRA index was used as risk assessment method. (Akyol \& Baykasoğlu, 2016). There are little number of studies that inspect machine scheduling and WMSD risks together. The human performance in industrial scheduling was inspected. It was defined that researches dealing human role in planning and scheduling need to be multi-disciplinary. In addition, it was reported that these studies need to be benefited from different disciplines like manufacturing, engineering, ergonomics, psychology, information and management science. A framework that aims to guide studies examining human in scheduling and manufacturing industry was developed (MacCarthy, Wilson, \& Crawford, 2001; Şenyiğit \& Atici, 2018).

Computer aided risk assessment instrument towards evaluation of WMSDs was presented. The instrument not only benefits from checklists, pictures and videos recorded during observation but also involves different risk assessment methods (Şenyiğit \& Atici, 2017a). Single machine scheduling problem under ergonomic risk factors was studied. OCRA index was used as risk assessment method and included to scheduling equations as a constant value (Şenyiğit \& Atici, 2017b; Şenyiğit \& Atici, 2018). It was presented that decision makers can not exactly determine the due-date and processing time due to insufficiencies in experience, judgment and knowledge. It proposes that there is an increasing interest towards fuzzy parameters with scheduling problem (Arık \& Toksarı, 2017).

Scheduling problem was tried to be close to real life case by forming increasing and decreasing parameters on processing time. Scheduling problem under job dependent learning effect was studied. Learning-curve approach was modelled as a repetitive function of manufacturing shift (Mosheiov \& Sidney, 2003). Learning effect and deterioration with single machine scheduling problem were studied. Deterioration and learning effect were defined as a function which depended on job starting time and position (J.-B. Wang, 2007), (J.-B. Wang, Huang, Wang, Yin, \& Wang, 2009). General learning effect with single machine scheduling problem was studied. A solution was proposed in which learning effect depended on total processing time and had a shortening effect on processing time (J.B. Wang, 2008). Single machine scheduling under non-linear deterioration and time dependent learning effect was studied. It was accepted that processing time prolongs in case of tardiness and shortens in case of learning (Toksarı, Oron, \& Güner, 2009). Past sequence dependent setup times with single machine scheduling problem was handled (Koulamas \& Kyparisis, 2010). Single machine scheduling with linear decreasing deterioration was studied (X.-R. Wang, Huang, \& Wang, 2011). Single machine scheduling with real time dependent and job dependent learning effect was tackled (Jiang, Chen, \& Kang, 2013). Unrelated parallel machine scheduling with job deterioration and source dependent processing time was inspected. Actual processing time was defined as the function of source different than starting time (Yin, Kang, Sun, Yue, \& Wang, 2014). Position dependent deterioration was studied. Weights were defined as position dependent and processing time was modelled as a function of basic processing time (Lu, Wang, \& Huang, 2015). Single machine scheduling based on total processing time and job positions was handled. Actual processing time was modelled with job position and total basic processing time (Lu et al., 2015). Oron studied on position dependent workloads and controllable process period (Oron, 2016). Toksari and Arık proposed single machine scheduling problem under fuzzy processing time with position dependent fuzzy learning effect (Toksari \& Arık, 2017). Koulamas studied on general due-date assignment with earliness and tardiness penalties (Koulamas, 2017).

In this study, a model which includes learning effect and WMSD risk factors have effect on process time was proposed. WMSD risk factors are considered as cumulative mean of risk value throughout shift. We proposed that schedules performance can be improve by balancing productivity and WMSD risk. It is aimed to decrease schedule mean of risk without increasing total basic process time. Total flow time and mean of risk were compared for obtained schedules. Balanced schedule total flow time and mean of risk is smaller than non-balanced Schedule. It was shown that handled problem is solvable at the polynomial time and total flow time can be improved by bringing balance between WMSD risks and productivity.

The rest of this article is organized as total flow time minimization problem on parallel machines with learning effect and WMSD risk factors in the second section, numerical results and discussion in the third section, conclusions and recommendations are presented in fourth section. 


\section{Material and Method}

\subsection{Scheduling problem with learning effect and WMSD risk factors}

In this paper, we propose a model to solve parallel machine problem under WMSD risk consideration. The imposed WMSD risks change according to machine, job type and technical activities number. By the WMSD consideration, we mean that job processing times are an increasing function of WMSD risk factor. WMSD risk factors have an increasing effect on processing times, for this reason WMSD risk factor included scheduling equation as deterioration rate. OCRA index method was selected as risk assessment technique, therefore, OCRA index provides an observation-based index value, which is related to the expected percentage of pathological cases among the working population and includes the cycle time which one of the candidate parameters that can be used in scheduling problems. Workstations improve continuously as a result of repeating the same or similar activities. Thus, the processing time of a job is shorter if it is scheduled later, rather than in the sequence. This phenomenon is known in the literature as a "learning effect". In this study, position based learning rate was used.

Job risk value is depend on cumulative average of risk values of previous jobs. Hereafter jobs risk value will be named as jobs deterioration rate. It is assumed that deterioration rate for each one of jobs should be different. Jobs processed on the machine shouldn't be completed within basic processing time due to the imposed ergonomic risks because of repetitive technical activities. According to this assumption if the job has the higher OCRA index value then its processing time will be larger.

OCRA index can be calculated by ratio between the foreseeable frequencies $(F F)$ of technical activities actions required the job and the reference frequency $(R F)$ of job. $F F$ is given by (NTC.60)/FCT (see Eq.(1)). NTC is the number of technical actions required to perform job during one cycle. $F C T$ is foreseeable duration of cycle time in seconds. $R F$ is given by $C F x P o_{m} x R e_{m} x A d_{\mathrm{m}} x F o_{m} x$ $\left(R c_{m} x D u_{m}\right)$. OCRA index is calculated for right and left body limp. The higher risk value is selected during the assessment. $C F$ is constant of frequency of technical actions per minute and equal to 30. $P o_{m}, R e_{m}, A d_{m}, F o_{m}, R c_{m}$ and Du are relatively postures, repetitiveness, additional, force, lack of recovery period and duration multiplier, respectively.

$$
O C R A=\frac{\frac{N T C 60}{F C T}}{k_{f} \times P o_{m} \times R e_{m} \times A d_{m} \times F o_{m} \times\left(R c_{m} \times D u_{m}\right)}
$$

We assume that $n$ is jobs number, $p_{j r}$ is actual processing time of job $j$ in the $r^{\text {th }}$ position in a sequence $S$. We use the learning effect model suggested by Biskup (Biskup, 1999). In this model, learning effect was represented by a $(\mathrm{a}<0)$ and it was formulated as a= $\log \alpha / \log 2$ where $\alpha$ is learning rate(Biskup, 1999). Biskup defined the actual processing time as $p_{j r}=p_{j} r^{a}$ where $p_{j}$ is the basic processing time of job $j$. In this paper, we propose actual processing time with learning effect and WMSD risk factors, simultaneously. We consider following models for the cumulative risk deterioration (CRD) (see Eq.(2)). OCRA risk assessment technique generates index value which is between 0 and 9 (Boenzi et al., 2013) thus $O C R A_{\max }$ equal to 9.

$$
C R D=1+\frac{\sum_{i=1}^{r} O C R A_{i}}{O C R A_{\max } \sum_{i=1}^{r} i} \quad(j=1, \ldots \ldots n)
$$

Process times and risk values for sample data set were given in Table 1. The data were obtained from observations done in electric household appliances manufacturing factory in Kayseri. In these observations, job risk assessment performed and jobs risk values were recorded. 20 different jobs were selected for scheduling. Jobs differed from each other in terms of number of technical activity, gender of employee and technical characteristics of job. The observation was performed during in normal shift and day time. The observation environment was company plant and no replacement was made in the layout. The basic processing times of jobs were taken from company's technical document and risk value of jobs ware gained by performing risk assessment. We assume that the learning rate is $80 \%$.

\begin{tabular}{|c|c|c|c|c|c|c|c|c|c|c|c|c|c|c|c|c|c|c|c|c|}
\hline Jobs & 1 & 2 & 3 & 4 & 5 & 6 & 7 & 8 & 9 & 10 & 11 & 12 & 13 & 14 & 15 & 16 & 17 & 18 & 19 & 20 \\
\hline Process Times & 8 & 7 & 7 & 8 & 9 & 9 & 10 & 13 & 15 & 16 & 18 & 20 & 15 & 15 & 19 & 19 & 19 & 21 & 40 & 48 \\
\hline Risk Values & 2.6 & 2.5 & 3.0 & 4.5 & 4.5 & 3.5 & 7.6 & 6.5 & 6.1 & 6.1 & 3.9 & 3.9 & 6.1 & 6.6 & 3.9 & 1.7 & 2.7 & 2.5 & 2.6 & 2.3 \\
\hline $\begin{array}{l}\text { OCRA Risk } \\
\text { Level }\end{array}$ & 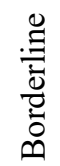 & 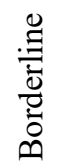 & 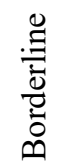 & $\frac{\mathscr{\Xi}}{\stackrel{\Xi}{\overline{0}}}$ & 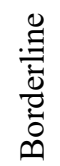 & $\frac{\mathscr{\Xi}}{\stackrel{\Xi}{\overline{0}}}$ & 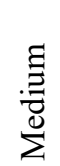 & $\stackrel{\Xi \Xi \Xi}{\Xi}$ & $\frac{\Xi}{\Xi}$ & $\frac{\Xi}{\Xi}$ & 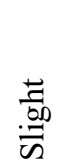 & $\begin{array}{l}\text { 苟 } \\
. \overline{=}\end{array}$ & $\stackrel{\Xi}{\Xi}$ & $\underset{\Xi}{\Xi}$ & 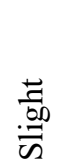 & $\begin{array}{l}\frac{0}{0} \\
\frac{\pi}{2} \\
\frac{0}{8} \\
\frac{0}{4}\end{array}$ & 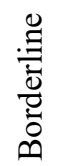 & 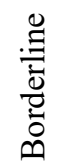 & 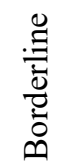 & 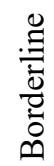 \\
\hline
\end{tabular}

Table 1. Jobs' process times and risk values 


\subsection{Total flow time minimization problem on parallel machines}

Total flow time minimization on parallel machines problem solution can be search in $m x n ! x n$ permutation and requires $O\left(n^{2}\right)$ computational efforts. Problem can be solved by transforming assignment problem. In spite of the fact that the computational effort remains $O\left(n^{4}\right)$, problem can be solve within more efficient time. We assume that $n$ is jobs number, $m$ is machine number and positional weight job $j$ is $\left(n_{i}-r+1\right)$ when it is assigned to position $r$ on machine $i$. Thus, the actual processing time of job $j$ processed on machine $i$ is $p_{i j r}=p_{j} r^{a}$ where $p_{j}$ is the basic processing time of job $j$. Objective of model is minimize to total flow time (see Eq.(3)). Only one job can be processed in one position on a machine (see Eq.(4)). Each job can only be processed in one position on a machine (see Eq.(5)). Let $x_{i j r}$ be a $0 / 1$ variable such that $x_{i j r}=1$ if job $j$ is scheduled in position $\mathrm{r}$ on machine $\mathrm{i}$, and $\mathrm{x}_{\mathrm{jr}}=0$ otherwise ((see Eq.(6))). The optimal total flow time on parallel machines is the solution of the following assignment problem:

$$
\begin{array}{lcr}
\min \sum_{i=1}^{m} \sum_{j=1}^{n} \sum_{r=1}^{n}\left(n_{i}-r+1\right) p_{i j r} x_{i j r} & \\
\sum_{j=1}^{n} x_{i j r}=1 & r=1, \ldots, n & i=1, \ldots, m \\
\sum_{i=1}^{m} \sum_{r=1}^{n} x_{i j r}=1 & j=1, \ldots, n \\
x_{i j r}=0 \quad \text { or } \quad 1, & j, r=1, \ldots, n & i=1, \ldots, m
\end{array}
$$

Total flow time minimization problem objective functions were rewrote for proposed deteriorating models. We consider following objective function for CRD (see Eq.(7)).

$$
\min \sum_{i=1}^{m} \sum_{j=1}^{n} \sum_{r=1}^{n}\left(n_{i}-r+1\right)\left(1+\frac{\sum_{i=1}^{r} O C R A_{i}}{\text { OCRA } \max ^{\prime} \sum_{i=1}^{r}}\right) p_{j} r^{a} x_{i j r}
$$

\section{Results and Discussion}

\begin{tabular}{|c|c|c|c|c|c|c|c|c|c|c|c|c|c|c|c|c|c|c|c|c|c|}
\hline \multirow{2}{*}{\multicolumn{2}{|c|}{ Positions }} & \multicolumn{10}{|c|}{ Machine -1 } & \multicolumn{10}{|c|}{ Machine -2 } \\
\hline & & 1 & 2 & 3 & 4 & 5 & 6 & 7 & 8 & 9 & 10 & 1 & 2 & 3 & 4 & 5 & 6 & 7 & 8 & 9 & 10 \\
\hline \multirow{2}{*}{ క্ } & Risk Value & 2.55 & 3.92 & 2.66 & 1.73 & 6.11 & 6.1 & 7.64 & 4.54 & 2.48 & 2.59 & 2.29 & 2.47 & 3.92 & 3.92 & 6.12 & 6.61 & 6.49 & 3.47 & 2.98 & 4.54 \\
\hline & Actual Time & 41.3 & 16.4 & 13.6 & 12.3 & 9.81 & 8.72 & 5.58 & 4.76 & 3.52 & 3.88 & 48.9 & 17.3 & 13.7 & 11.8 & 9.23 & 8.75 & 7.22 & 4.73 & 3.52 & 3.91 \\
\hline
\end{tabular}

We provide 20-job example in order show how the schedule changes under WMSD consideration and why WMSD risks should be taken into account in parallel machine scheduling. The sample data set given in Table 1 was used to solve the CRD model. The models were coded using LINGO software. Jobs' sequence, risk value, process time and actual times are as shown in Table 2.

Table 2. Jobs'sequence and actual times for S schedule

Operators are exposed medium risk level while performing jobs 7, 8, 9, 10, 13, 14. Figure-1 shows risk levels along the schedule $S$ for each machine. Medium risk level painted with red.
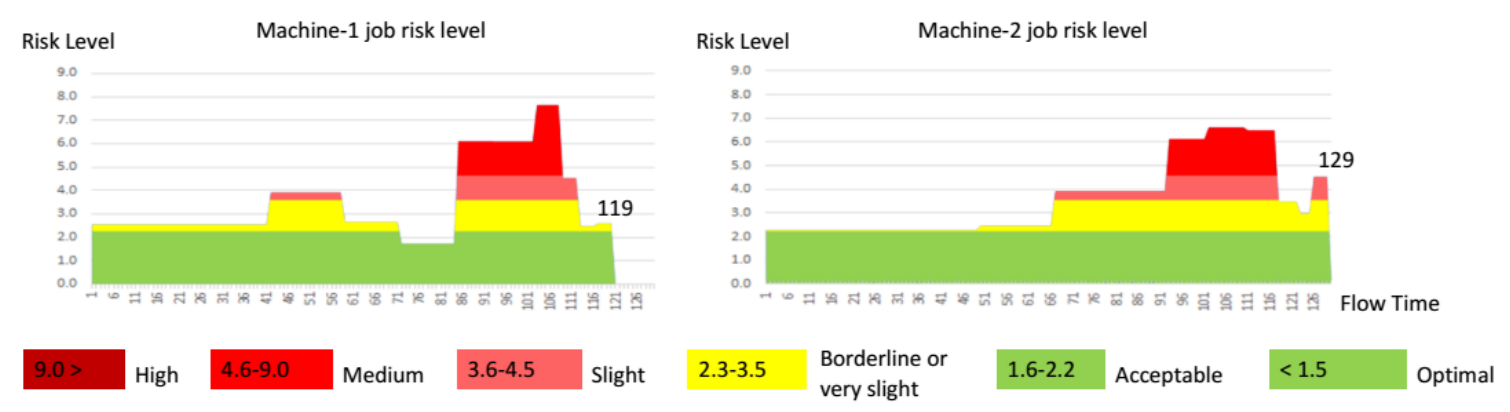

Figure 1. Job risk levels for $S$ schedule with learning rate and CRD

Jobs sequence is respectively $\mathrm{j}-19, \mathrm{j}-12, \mathrm{j}-17, \mathrm{j}-16, \mathrm{j}-13, \mathrm{j}-7, \mathrm{j}-5, \mathrm{j}-2, \mathrm{j}-1$ on machine- 1 and $\mathrm{j}-20, \mathrm{j}-18, \mathrm{j}-15, \mathrm{j}-11, \mathrm{j}-9, \mathrm{j}-14, \mathrm{j}-8, \mathrm{j}-$ $6, \mathrm{j}-3, \mathrm{j}-4$ on machine-2. Figure-2 shows jobs sequence with CRD and learning rate. 


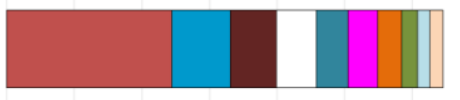

Figure 2. Job sequences for each machine with CRD and learning rate.

In order to the balance between productivity and WMSD risk, jobs' risk level balanced. FCT values of jobs which have medium risk (jobs $7,8,9,10,13,14$ ) are increased until the risk value is slight. The value of sacrifice from the FCT value was met by decreasing the FCT value of the lower jobs (jobs 1, 2, 16, 17, 18, 19, 20). Thus, total basic process time remained same value. Jobs basic process time and risk value recalculated. Balanced Cumulative Risk Deterioration Model (BCRD) solved by using Lingo software with new basic process times and risk values. Jobs' sequence, risk value, process time and actual times for BCRD are as shown in Table 3.

Table 3. Balanced jobs'sequence and actual times for S'schedule

\begin{tabular}{|c|c|c|c|c|c|c|c|c|c|c|c|c|c|c|c|c|c|c|c|c|c|}
\hline \multirow{2}{*}{\multicolumn{2}{|c|}{ Positions }} & \multicolumn{10}{|c|}{ Machine -1 } & \multicolumn{10}{|c|}{ Machine -2 } \\
\hline & & 1 & 2 & 3 & 4 & 5 & 6 & 7 & 8 & 9 & 10 & 1 & 2 & 3 & 4 & 5 & 6 & 7 & 8 & 9 & 10 \\
\hline $\mathfrak{z}$ & Risk Value & 3.51 & 4.54 & 4.54 & 3.92 & 3.92 & 3.55 & 4.46 & 2.98 & 4.54 & 3.02 & 3.54 & 4.41 & 4.54 & 3.92 & 4.46 & 3.53 & 3.46 & 3.47 & 4.54 & 3.31 \\
\hline & Actual Time & 31.8 & 14.5 & 12.7 & 11.2 & 10.4 & 8.62 & 6.58 & 4.18 & 3.54 & 2.92 & 31.7 & 15.2 & 12.7 & 11.2 & 9.8 & 8.05 & 5.46 & 4.18 & 3.54 & 2.92 \\
\hline
\end{tabular}

None of operators are exposed medium risk level while performing jobs. Figure-3 shows risk levelS along the schedule $S^{\prime}$ for each machine.
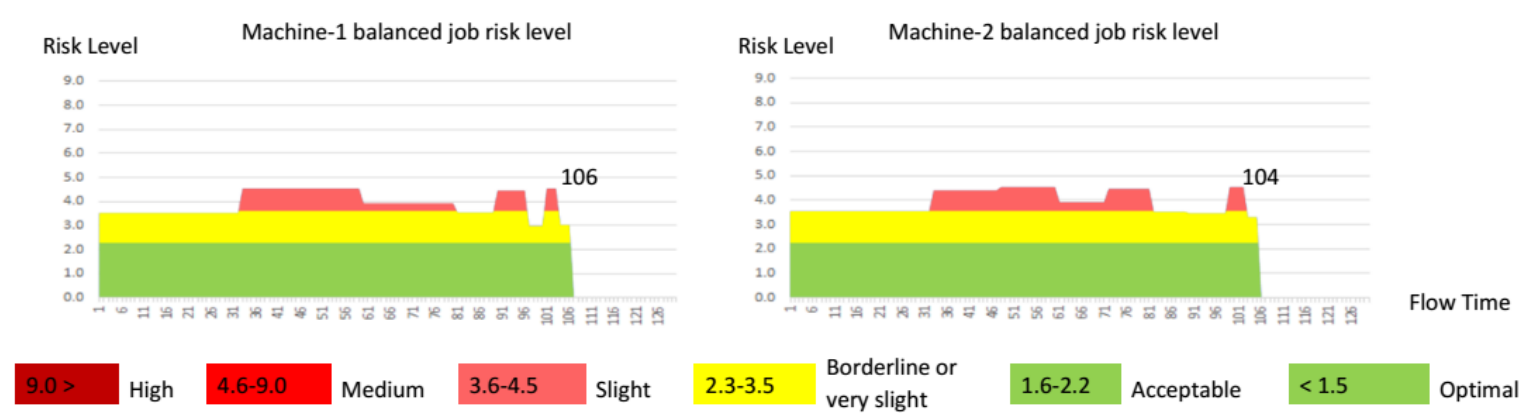

Figure 3. Job risk level for $S^{\prime}$ schedule with learning rate and CRD

Jobs sequence is respectively $j-19, j-13, j-10, j-15, j-17, j-7, j-3, j-5, j-1$ on machine-1 and $j-20, j-14, j-9, j-11, j-8, j-18, j-16, j-$ $6, j-4, j-2$ on machine-2. Figure-4 shows jobs sequence with BCRD and learning rate.

Balanced job sequence on machine-1
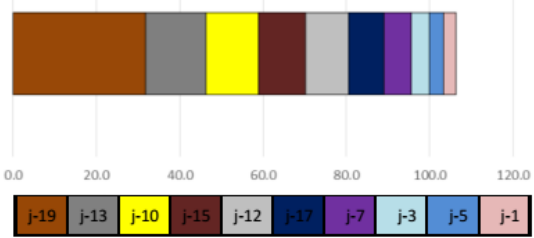

Balanced job sequence on machine-2

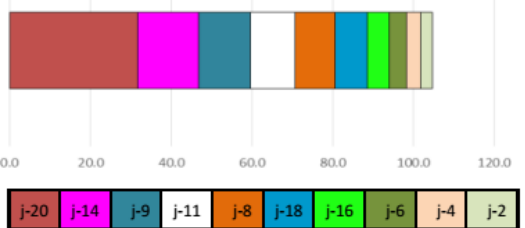

Figure 4. Job sequences for each machine with learning rate and BCRD.

The average of the schedule $S$ risk level is 4.1 and total flow time is 248 with provided process time and risk value. After the balancing the average of the schedule $S$ ' risk level is 3.9 and total flow time is 210 with balanced process time and risk value. It was shown that improvement in total flow time can be achieved by balancing between WMSD risks and productivity. 


\section{Conclusions and Recommendations}

In this study, parallel machine scheduling problem under WMSD consideration was introduced. WMSD risks were modeled with cumulative mean risk value of OCRA index which effective throughout the shift. Due to NP-Hard structure of parallel machine scheduling problems with WMSD considerations and learning rate, the solution cannot be found always. Handled problem was solved by transforming assignment problem. In spite of the fact that the computational effort remains $O\left(n^{4}\right)$, problem was solved within more efficient time. S schedule that contain sequence of jobs and actual times gained. Thus, jobs have medium risk level and actual times can be determined.

In order to the balance between productivity and WMSD risk, jobs' FCT values were changed. It is aimed to decrease schedule mean risk value without increasing total basic process time. To reduce risk level FCT values of jobs which have medium or high risk level were increased. The value of sacrifice from the FCT was compensating from job which have optimal, acceptable, borderline or slight risk level. Process time and risk values were recalculated. BCRD model was solved and S' schedule was obtained. The schedule $\mathrm{S}$ average risk level and total flow time are respectively 4.1 and 248 for provided process times with CRD and learning rate. The schedule S' average risk level and total flow time are respectively is 3.9 and 210 with BCRD and learning rate. Total flow time and mean of risk belong to BCRD model is smaller than CRD model. Therefore parallel machine schedule with WMSD risk consideration and learning rate is solvable at the polynomial time and total flow time can be improve by bringing balance between WMSD risks and productivity.

Innovation of this study is to model for the first time in a parallel machine scheduling problem including WMSD risk and to show that handled problem is solvable at the polynomial time. Also it is shown that bringing balance between WMSD risks and productivity can improve total flow time.

Finally, the benefits of the proposed model are that may allow more accurate production planning, may let to monitoring employee tempo with smart individual protective clothing. Models can apply to different scheduling problems; different risk assessment methods can be taken into account instead of OCRA index as further works.

\section{Acknowledge}

This study is supported by Erciyes University Scientific Research Projects Unit in the scope of "Scheduling under Ergonomic Risk Factors" coded FDK-2017-7265 project.

\section{References}

Akyol, Ş. D., \& Baykasoğlu, A. (2016). ErgoALWABP: a multiple-rule based constructive randomized search algorithm for solving assembly line worker assignment and balancing problem under ergonomic risk factors. Journal of Intelligent Manufacturing. doi: 10.1007/s10845-016-1246-6

Antwi-Afari, M. F., Li, H., Edwards, D. J., Pärn, E. A., Seo, J., \& Wong, A. Y. L. (2017). Biomechanical analysis of risk factors for work-related musculoskeletal disorders during repetitive lifting task in construction workers. Automation in Construction, 83, $41-$ 47. doi: https://doi.org/10.1016/j.autcon.2017.07.007

Arık, O. A., \& Toksarı, M. D. (2017). Multi-objective fuzzy parallel machine scheduling problems under fuzzy job deterioration and learning effects. International Journal of Production Research, 1-18. doi: 10.1080/00207543.2017.1388932

Aven, T. (2016). Risk assessment and risk management: Review of recent advances on their foundation. European Journal of Operational Research, 253(1), 1-13. doi: https://doi.org/10.1016/j.ejor.2015.12.023

Battini, D., Glock, C. H., Grosse, E. H., Persona, A., \& Sgarbossa, F. (2015). Ergo-Lot-Sizing: Considering Ergonomics in Lot-Sizing Decisions. IFAC-PapersOnLine, 48(3), 326-331. doi: https://doi.org/10.1016/j.ifacol.2015.06.102

Biskup, D. (1999). Single-machine scheduling with learning considerations. European Journal of Operational Research, 115(1), 173178. doi: https://doi.org/10.1016/S0377-2217(98)00246-X

Boenzi, F., Digiesi, S., Mossa, G., Mummolo, G., \& Romano, V. A. (2013). Optimal Break and Job Rotation Schedules of High Repetitive - Low Load Manual Tasks in Assembly Lines: an OCRA - Based Approach. IFAC Proceedings Volumes, 46(9), 18961901. doi: https://doi.org/10.3182/20130619-3-RU-3018.00625

Fulmer, S., Buchholz, B., Scribani, M., \& Jenkins, P. (2017). Musculoskeletal Disorders in Northeast Lobstermen. Safety and Health at Work, 8(3), 282-289. doi: https://doi.org/10.1016/j.shaw.2016.12.004 
Hignett, S., \& McAtamney, L. (2000). Rapid Entire Body Assessment (REBA). Applied Ergonomics, 31(2), 201-205. doi: https://doi.org/10.1016/S0003-6870(99)00039-3

Jiang, Z., Chen, F., \& Kang, H. (2013). Single-machine scheduling problems with actual time-dependent and job-dependent learning effect. European Journal of Operational Research, 227(1), 76-80. doi: https://doi.org/10.1016/j.ejor.2012.12.007

Koulamas, C. (2017). Common due date assignment with generalized earliness/tardiness penalties. Computers \& Industrial Engineering, 109, 79-83. doi: https://doi.org/10.1016/j.cie.2017.04.040

Koulamas, C., \& Kyparisis, G. J. (2010). Single-machine scheduling problems with past-sequence-dependent delivery times. International Journal of Production Economics, 126(2), 264-266. doi: https://doi.org/10.1016/j.ijpe.2010.03.016

Lu, Y.-Y., Wang, J.-J., \& Huang, X. (2015). Scheduling jobs with position and sum-of-processing-time based processing times. Applied Mathematical Modelling, 39(14), 4013-4021. doi: 10.1016/j.apm.2014.12.021

MacCarthy, B. L., Wilson, J. R., \& Crawford, S. (2001). Human performance in industrial scheduling: A framework for understanding. Human Factors and Ergonomics in Manufacturing \& Service Industries, 11(4), 299-320. doi: doi:10.1002/hfm.1016

McAtamney, L., \& Nigel Corlett, E. (1993). RULA: a survey method for the investigation of work-related upper limb disorders. Applied Ergonomics, 24(2), 91-99. doi: https://doi.org/10.1016/0003-6870(93)90080-S

Micheli, G. J. L., \& Marzorati, L. M. (2018). Beyond OCRA: Predictive UL-WMSD risk assessment for safe assembly design. International Journal of Industrial Ergonomics, 65, 74-83. doi: https://doi.org/10.1016/j.ergon.2017.07.005

Mosheiov, G., \& Sidney, J. B. (2003). Scheduling with general job-dependent learning curves. European Journal of Operational Research, 147(3), 665-670. doi: https://doi.org/10.1016/S0377-2217(02)00358-2

Occhipinti, E. (1998). OCRA: a concise index for the assessment of exposure to repetitive movements of the upper limbs. Ergonomics, 41(9), 1290-1311. doi: 10.1080/001401398186315

Occhipinti, E., \& Colombini, D. (2016). A toolkit for the analysis of biomechanical overload and prevention of WMSDs: Criteria, procedures and tool selection in a step-by-step approach. International Journal of Industrial Ergonomics, 52, 18-28. doi: https://doi.org/10.1016/j.ergon.2015.08.001

Oron, D. (2016). Scheduling controllable processing time jobs with position-dependent workloads. International Journal of Production Economics, 173, 153-160. doi: https://doi.org/10.1016/j.ijpe.2015.12.014

Otto, A., \& Scholl, A. (2011). Incorporating ergonomic risks into assembly line balancing. European Journal of Operational Research, 212(2), 277-286. doi: 10.1016/j.ejor.2011.01.056

Schaub, K., Caragnano, G., Britzke, B., \& Bruder, R. (2013). The European Assembly Worksheet. Theoretical Issues in Ergonomics Science, 14(6), 616-639. doi: 10.1080/1463922X.2012.678283

Şenyiğit, E., \& Atici, U. (2017a). Computer-aided work related musculoskeletal disorder risk assessment tool: WMSD-RA. Paper presented at the International Symposium on Industry 4.0 and Applications, Karabuk, Turkey.

Şenyiğit, E., \& Atici, U. (2017b). Ergo-Scheduling. New Trends and Issues Proceedings on Humanities and Social Sciences, 4(10), 208217.

Şenyiğit, E., \& Atici, U. (2018). Scheduling with Job Dependent Learning Effect and Ergonomic Risk Deterioration. Proceedings of the 2018 2nd International Symposium on Multidisciplinary Studies and Innovative Technologies (ISMSIT), Ankara, Turkey, 63-66.

Toksari, M. D., \& Arık, O. A. (2017). Single machine scheduling problems under position-dependent fuzzy learning effect with fuzzy processing times. Journal of Manufacturing Systems, 45, 159-179. doi: https://doi.org/10.1016/j.jmsy.2017.08.006

Toksarı, M. D., Oron, D., \& Güner, E. (2009). Single machine scheduling problems under the effects of nonlinear deterioration and time-dependent learning. Mathematical and Computer Modelling, 50(3-4), 401-406. doi: 10.1016/j.mcm.2009.05.026

Occupational Health And Safety (2012).

TSE. (2011). Safety of machinery - Human physical performance Part-5: Risk assessment for repetitive handling at high frequency (Vol. TS EN 1005-5). Ankara.

Wang, J.-B. (2007). Single-machine scheduling problems with the effects of learning and deterioration. Omega, 35(4), 397-402. doi: https://doi.org/10.1016/j.omega.2005.07.008

Wang, J.-B. (2008). Single-machine scheduling with general learning functions. Computers \& Mathematics with Applications, 56(8), 1941-1947. doi: https://doi.org/10.1016/j.camwa.2008.04.019

Wang, J.-B., Huang, X., Wang, X.-Y., Yin, N., \& Wang, L.-Y. (2009). Learning effect and deteriorating jobs in the single machine scheduling problems. Applied Mathematical Modelling, 33(10), 3848-3853. doi: https://doi.org/10.1016/j.apm.2009.01.004

Wang, X.-R., Huang, X., \& Wang, J.-B. (2011). Single-machine scheduling with linear decreasing deterioration to minimize earliness penalties. Applied Mathematical Modelling, 35(7), 3509-3515. doi: 10.1016/j.apm.2011.01.005

Yin, N., Kang, L., Sun, T.-C., Yue, C., \& Wang, X.-R. (2014). Unrelated parallel machines scheduling with deteriorating jobs and resource dependent processing times. Applied Mathematical Modelling, 38(19), 4747-4755. doi: https://doi.org/10.1016/j.apm.2014.03.022 